\title{
Symbolic Harmonic Analysis of Quartz Crystal Oscillators
}

\author{
Nicolas Ratier, Mickaël Bruniaux, and Rémi Brendel \\ Institut FEMTO-ST, département LPMO, CNRS UMR 6174, 32 avenue de l'Observatoire, F-25044 Besançon, France \\ Jérôme Delporte \\ Centre National d'Etudes Spatiales, Toulouse, France
}

\begin{abstract}
The Nonlinear Dipolar Method is dedicated to the simulation of quartz crystal oscillator with high quality factor. In this method, the oscillators is considered as a resonator connected across an amplifier that behaves like a nonlinear dipole whose impedance evaluated at resonator's frequency depends on the current amplitude. This dipole allows us to compute very quickly the behavior of the oscillator. The computation time of the dipolar impedance by Spice is of the order of a few seconds. To gain one order of magnitude in the simulation time of the oscillator, this paper propose a modification of the Nonlinear Dipolar Method by changing the dipolar impedance Spice's calculation, that is the most time consuming part of the program, by a system of equations obtained through a symbolic manipulation of the circuit equations.
\end{abstract}

\section{INTRODUCTION}

We develop a method of analysis of quartz crystal oscillators with very high quality factor. The ultimate aim is to get a real time analysis of this type of oscillators.

In a previous series of articles [1][2][3], we have developed a method, called Nonlinear Dipolar Method, which allows to simulate quartz oscillator very efficiently. The calculation needs only a few seconds for a Colpitts oscillator.

The most time consuming part of the dipolar method is the calculation of the dipolar impedance $R_{d}(a)$ and $L_{d}(a)$ where $a$ is the loop current amplitude. The idea is to replace the previously performed calculation through a series of Spice analysis, by a symbolic analyses of the circuit.

First, The circuit equations are generated by using the Modified Nodal Method (MNM). Then, a symbolic harmonic analysis of these equations is performed. Eventually, a system of nonlinear equations representing $R_{d}(a)$ et $L_{d}(a)$ and rapidly resolvable is generated.

We start by recalling briefly in this paper the dipolar method and in particular the calculation of $R_{d}(a)$ et $L_{d}(a)$. We, then, present the new method that replaces the dipolar impedance calculation by a symbolic harmonic analysis of the equations.

\section{Nonlinear Dipolar Method}

The nonlinear dipolar method is based on the fact that the current through the motional branch of the quartz is almost perfectly sinusoidal (because of its very high $Q$ ). Moreover, the oscillator frequency is mainly determined by the resonant frequency of the resonator.
Following [3], these two remarks permit to consider the whole amplifier part (and capacity $C_{p}$ ) as a nonlinear dipole $\left(R_{d}, L_{d}\right)$ that depends only on the current amplitude as shown in Fig. 1 right. In the following, by misuse of language, we will call "resonator" the motional branch of the resonator, and "amplifier" the amplifier in parallel with the capacitance $C_{p}$.

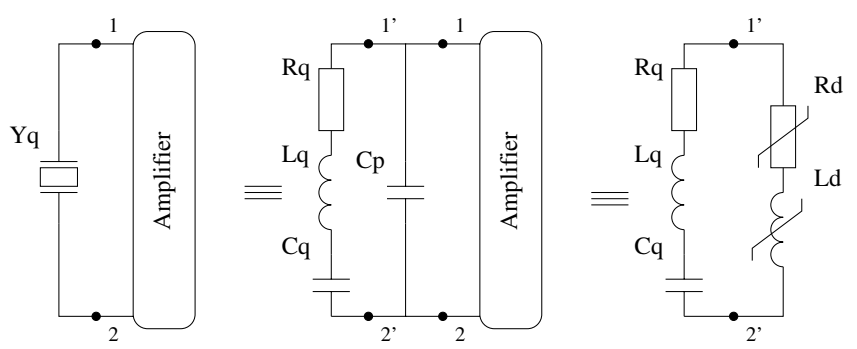

Fig. 1. Dipolar representation

The resonator is regarded as an impedance strongly varying with the frequency and slightly with amplitude (because of the isochronism defect) while the amplifier is considered to be a nonlinear dipole whose impedance strongly depends on the current amplitude and weakly varying with frequency.

The knowledge of $R_{d}(a)$ and $L_{d}(a)$ allows us to compute very quickly the steady state and transient amplitude and frequency. The steady state amplitude $a_{0}$ is obtained by solving the implicit nonlinear Eq. (1)

$$
R_{q}+R_{d}(a)=0
$$

The steady state frequency $\omega_{0}$ of the oscillation is given by Eq. (2).

$$
\omega_{0}^{2}=\omega_{q}^{2}\left(1-\frac{L_{d}\left(a_{0}\right)}{L_{q}}\right)
$$

The current through the motional branch of the quartz is written under the form $i(t)=a(t) \cos \left(\omega_{q} t+\phi(t)\right)$ where the amplitude $a(t)$ and phase $\phi(t)$ are slowly varying functions of time. The transient amplitude and frequency are obtained by solving the first order differential equations $(3,4)$.

$$
\begin{aligned}
\frac{d a}{d t} & =-\frac{a}{2 L_{q}}\left(R_{q}+R_{d}(a)\right) \\
\frac{d \phi}{d t} & =-\frac{L_{d}(a) \omega_{q}}{2 L_{q}}
\end{aligned}
$$




\section{Spice COMPUTATION OF $R_{d}$ AND $L_{d}$}

The determination of the equivalent single port network for the amplifier is obtained by replacing the resonator with a sinusoidal current source of amplitude $a$ and frequency equal to the resonator's frequency $f_{q}$ as shown in Fig. 2 .

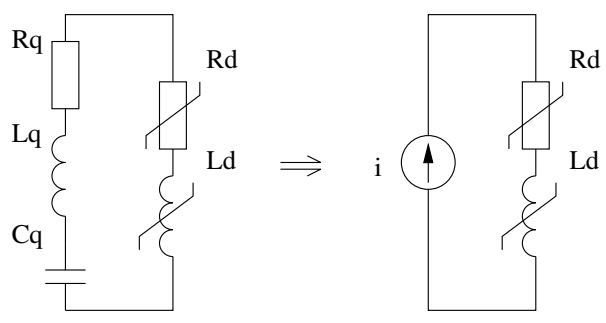

Fig. 2. Amplifier impedance determination

Then, a set of Spice transient analyses are performed at various amplitudes. Next, a Fourier analysis of the voltage across the amplifier is computed for each current amplitude when the steady state is reached.

It is thus possible to calculate the equivalent resistance $R_{d}$ and inductance $L_{d}$ as a function of the current amplitude (The subscript "d" stands for Dipole). Figs. 3 and 4 show $R_{d}$ and $L_{d}$ as a function of the current amplitude $a$ for the Colpitts oscillator.

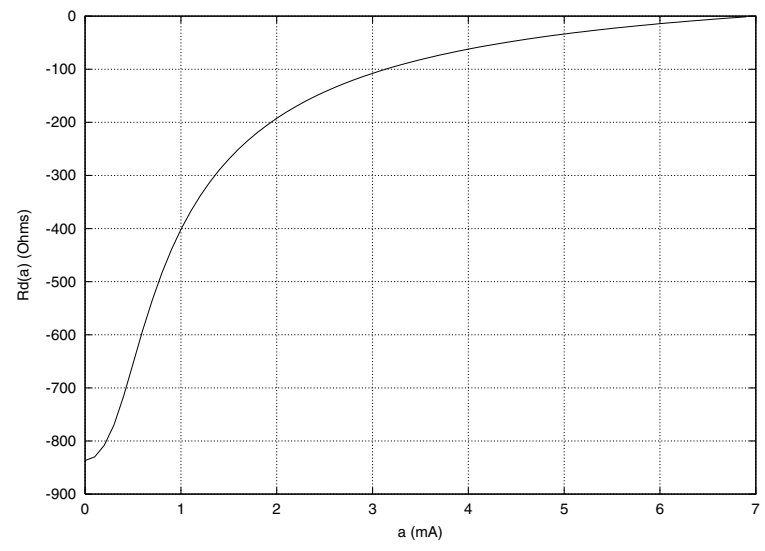

Fig. 3. Amplifier resistance $R_{d}(a)$

The computation time of the curves $R_{d}(a)$ and $L_{d}(a)$ is of the order of a few seconds. At each parameter change, for example a variation of resistance value, the computation must be redone. Our aim is to reduce the computation time for the curves $R_{d}(a)$ and $L_{d}(a)$ to a few milliseconds. This should allow to see, in real time, the influence of each circuit component on the behavior of the oscillator.

\section{Symbolic COMPUtation of $R_{d}$ AND $L_{d}$}

Our aim is to be able to calculate the curves $R_{d}(a)$ and $L_{d}(a)$ as fast as possible for a given oscillator. The idea

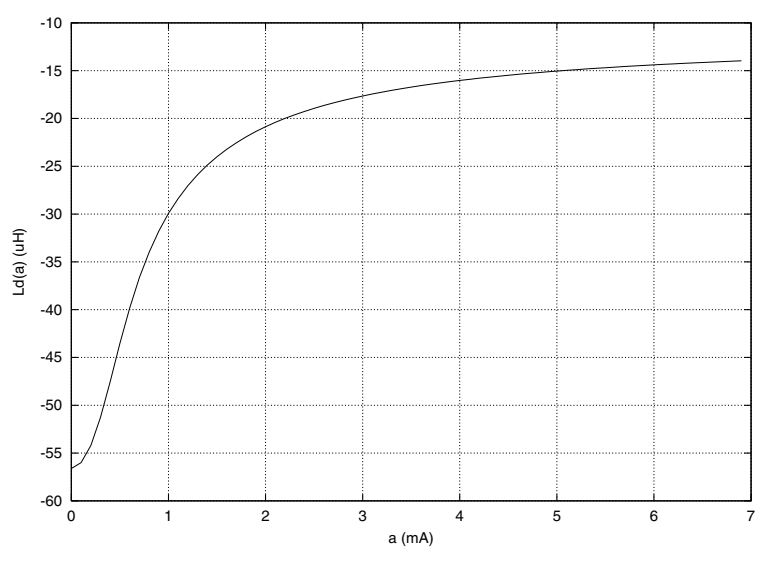

Fig. 4. Amplifier inductance $L_{d}(a)$

consists in replacing the previous Spice simulation by a symbolic analysis of the circuit generating a system of equations rapidly resolvable.

The equation setting is carried out in the following way. First the motional part of the quartz is replaced by a sinusoidal current source of amplitude $a$ and frequency $f_{q}$. This step is identical to the previous one. In addition, it ensures periodic signals in the whole circuit.

Then, the system of equations that models the electronic circuit is done symbolically by the Modified Nodal Method (MNM) [4]. If the circuit contains $n$ nodes and $n_{v}$ voltagedefined branches, then the $n-1$ nondatum node voltages and the $n_{v}$ voltage-defined branch currents become the unknown circuit variables. The set of modified nodal equations are obtained by applying the KCL (Kirchhoff's Current Law) to each nondatum node and including the $n_{v}$ voltagedefined branch relations.

The circuit equations are, in general, a system of algebraic-differential equations of the form (5).

$$
\mathbf{f}(\mathbf{x}, \dot{x}, \mathbf{t})=\mathbf{0}
$$

where $\mathbf{x}$ is the vector of the unknown circuit variables, $\mathbf{x}$ is the time-derivative of $\mathbf{x}$, and $\mathbf{f}$ is, in general, a nonlinear operator.

We assume a solution of the form (6) where $\mathbf{a}_{\mathbf{0}}, \mathbf{a}_{\mathbf{k}}$ and $\mathbf{b}_{\mathbf{l}}$ are constant vectors, only function of the circuit elements. The constants $(K, L)$ depend on the degree of the approximation chosen.

$$
\mathbf{x}=\mathbf{a}_{\mathbf{0}}+\sum_{\mathbf{k}=\mathbf{1}}^{\mathbf{K}} \mathbf{a}_{\mathbf{k}} \cos \mathbf{k} \omega \mathbf{t}+\sum_{\mathbf{l}=\mathbf{1}}^{\mathbf{L}} \mathbf{b}_{\mathbf{l}} \sin \mathbf{l} \omega \mathbf{t}
$$

By substituting (6) into the given differential equation (5), it is possible to write the system under the form (7). The constant vectors $\mathbf{a}_{\mathbf{0}}^{\prime}, \mathbf{a}_{\mathbf{m}}^{\prime}, \mathbf{b}_{\mathbf{n}}^{\prime}$ are function of the constant vectors 
$\mathbf{a}_{\mathbf{0}}, \mathbf{a}_{\mathbf{k}}$ and $\mathbf{b}_{\mathbf{l}}$. In the general case, $M \gg K$ and $N \gg L$.

$$
\mathbf{a}_{\mathbf{0}}^{\prime}+\sum_{\mathbf{m}=\mathbf{1}}^{\mathbf{M}} \mathbf{a}_{\mathbf{m}}^{\prime} \cos \mathbf{m} \omega \mathbf{t}+\sum_{\mathbf{n}=\mathbf{1}}^{\mathbf{N}} \mathbf{b}_{\mathbf{n}}^{\prime} \sin \mathbf{n} \omega \mathbf{t}=\mathbf{0}
$$

The system (7) must be verified for all $t$, that leads to the nonlinear system of equations (8) for the constant vectors $\mathbf{a}_{\mathbf{0}}, \mathbf{a}_{\mathbf{k}}$ and $\mathbf{b}_{\mathbf{l}}$. The space dimension of $\mathbf{F}$ depends, among other things, on the degree of the approximation chosen and is in general much bigger than those of $\mathbf{f}$. The system of equation (8) can be seen as a kind of Fourier Transform of the system (5).

$$
\mathbf{F}\left(\mathbf{a}_{\mathbf{0}}, \mathbf{a}_{\mathbf{1}}, \ldots, \mathbf{a}_{\mathbf{m}}, \mathbf{b}_{\mathbf{1}}, \ldots, \mathbf{b}_{\mathbf{n}}\right)=\mathbf{0}
$$

Even in the case of a circuit of about 30 elements, the nonlinear system (8) can be solved numerically very quickly (ms). The amplitude $a$ of the sinusoidal current source is a parameter of the system. A simple calculation on the scheme Fig. (2 right) shows that we can obtain $R_{d}(a)$ and $L_{d}(a)$ from the solution of equation $(9,10)$, with $\left(k, k^{\prime}\right)$ respectively the number of nodes $\left(1^{\prime}, 2^{\prime}\right)$.

$$
\begin{aligned}
& R_{d}(a)=\frac{b_{k}-b_{k^{\prime}}}{a} \\
& L_{d}(a)=\frac{a_{k}-a_{k^{\prime}}}{a \omega_{q}}
\end{aligned}
$$

The passage from the form (5) to that of (8) is performed by joining (or putting together) progressively the different terms. The addition and multiplication cases are trivials. The addition of two unknowns is also trivial and does not increase the order of the series.

The multiplication of two unknowns (i.e. Fourier series) call out a sum of terms in $\cos k \omega t \times \cos l \omega t$, $\cos k \omega t \times \sin l \omega t$ et $\sin k \omega t \times \sin l \omega t$. These products transform to $\cos (k+l) \omega t, \sin (k+l) \omega t, \cos (k-l) \omega t$ and $\sin (k-l) \omega t$ by classical trigonometric formulas. Consequently, each multiplication between unknowns progressively increases the degree of the series. Moreover, the high frequency terms intervene in the expression with low frequencies. One should then keep all the terms through the calculation.

\section{HOW TO MANAGE THE NONLINEAR COMPONENT ?}

The semiconductor components deal with nonlinear terms in the constitutive equations. As an example, the EbersMoll static model [5] of the bipolar transistor involves the exponential function of the voltage difference.

$$
\begin{aligned}
I_{B E} & =\frac{I_{S}}{\beta_{F}}\left(e^{q V_{B E} / k T}-1\right) \\
I_{B C} & =\frac{I_{S}}{\beta_{R}}\left(e^{q V_{B C} / k T}-1\right) \\
I_{C E} & =I_{S}\left(e^{q V_{B E} / k T}-e^{q V_{B C} / k T}\right)
\end{aligned}
$$

The main problem is to express all the functions, for example the exponential function

$\exp \left(a_{0}+a_{1} \cos \omega t+b_{1} \sin \omega t+a_{2} \cos 2 \omega t+\cdots\right)$, in the form of a Fourier series $a_{0}^{\prime}+a_{1}^{\prime} \cos \omega t+b_{1}^{\prime} \sin \omega t+$ $a_{2}^{\prime} \cos 2 \omega t+\cdots$. Thereafter, this transform will be called "to harmonize" a function. In the next section, it will be shown how to harmonize all the common functions : the transcendental functions, the inverse of a function and the composite functions.

\section{HARMONIZATION OF TRANSCENDENTAL PRIMARY FUNCTIONS}

Transcendental primary functions are the functions exp, cos, sin. We also include improperly the functions cosh and $\sinh$. Let $\alpha$ and $\beta$ be :

$$
\begin{aligned}
\alpha & =a_{m} \cos m \omega t \text { or } \alpha=b_{m} \sin m \omega t \\
\beta & =\sum_{k \neq m} a_{k} \cos k \omega t+\sum_{k \neq m} b_{k} \sin k \omega t
\end{aligned}
$$

Let us recall the addition formulas of function for completeness

$$
\begin{aligned}
\exp (\alpha+\beta) & =\exp \alpha \exp \beta \\
\cos (\alpha+\beta) & =\cos \alpha \cos \beta-\sin \alpha \sin \beta \\
\sin (\alpha+\beta) & =\sin \alpha \cos \beta+\cos \alpha \sin \beta \\
\cosh (\alpha+\beta) & =\cosh \alpha \cosh \beta+\sinh \alpha \sinh \beta \\
\sinh (\alpha+\beta) & =\sinh \alpha \cosh \beta+\cosh \alpha \sinh \beta
\end{aligned}
$$

Consequently, recursive application of the latter equations (16 to 20) shows that to harmonize the transcendental primary functions, it is sufficient to know how to harmonize the following functions:

$$
\begin{array}{ll}
\exp (z \cos \theta) & \exp (z \sin \theta) \\
\cos (z \cos \theta) & \cos (z \sin \theta) \\
\sin (z \cos \theta) & \sin (z \sin \theta) \\
\cosh (z \cos \theta) & \cosh (z \sin \theta) \\
\sinh (z \cos \theta) & \sinh (z \sin \theta)
\end{array}
$$

The harmonic expansion of $\exp (z \cos \theta)$ and $\exp (z \sin \theta)$ are well known. There are expressed in Fourier series, whose coefficients are Bessel functions $I_{k}(z)$ of the second kind and of integer order [6].

$$
\begin{aligned}
\exp (z \cos \theta)=I_{0}(z) & +2 \sum_{k=1}^{\infty} I_{k}(z) \cos (k \theta) \\
\exp (z \sin \theta)=I_{0}(z) & +2 \sum_{k=1}^{\infty}(-)^{k} I_{2 k+1}(z) \sin \{(2 k+1) \theta\} \\
& +2 \sum_{k=1}^{\infty}(-)^{k} I_{2 k}(z) \cos (2 k \theta)
\end{aligned}
$$


The harmonic expansion of $\cos (z \cos \theta)$ and $\sin (z \sin \theta)$ are expanded in Fourier series, whose coefficients are Bessel functions $J_{k}(z)$ of the first kind and of integer order [6].

$$
\begin{aligned}
\cos (z \cos \theta) & =J_{0}(z)+2 \sum_{k=1}^{\infty}(-)^{k} J_{2 k}(z) \cos (2 k \theta) \\
\cos (z \sin \theta) & =J_{0}(z)+2 \sum_{k=1}^{\infty} J_{2 k}(z) \cos (2 k \theta) \\
\sin (z \cos \theta) & =2 \sum_{k=1}^{\infty}(-)^{k} J_{2 k+1}(z) \cos \{(2 k+1) \theta\} \\
\sin (z \cos \theta) & =2 \sum_{k=1}^{\infty} J_{2 k+1}(z) \sin \{(2 k+1) \theta\}
\end{aligned}
$$

The harmonic expansion of $\cosh (z \cos \theta)$ and $\sinh (z \sin \theta)$ are calculated from the expansion of the exponential. Note the symmetry of cos, sin equations and those of cosh, sinh.

$$
\begin{aligned}
\cosh (z \cos \theta) & =I_{0}(z)+2 \sum_{k=1}^{\infty} I_{2 k}(z) \cos (2 k \theta) \\
\cosh (z \sin \theta) & =I_{0}(z)+2 \sum_{k=1}^{\infty}(-)^{k} I_{2 k}(z) \cos (2 k \theta) \\
\sinh (z \cos \theta) & =2 \sum_{k=1}^{\infty} I_{2 k+1}(z) \cos \{(2 k+1) \theta\} \\
\sinh (z \sin \theta) & =2 \sum_{k=1}^{\infty}(-)^{k} I_{2 k+1}(z) \sin \{(2 k+1) \theta\}
\end{aligned}
$$

All the previous expansions converge well with a few number of terms (typically 3 ) when $z$ is less than 1 .

\section{HARMONIZATION OF THE OTHER FUNCTIONS}

For all the other transcendental function (log, tan, arccos, ...) one proceeds by introducing a supplementary unknown to the equations system. This transforms the initial differential equation system Eq. (5) into an algebraic-differential system. This handling is trivial as shown in the following example

$$
\cdots+\tan V_{1}+\cdots+\arccos V_{2}+\cdots=0
$$

becomes

$$
\begin{aligned}
\cdots+H_{1}+\cdots+H_{2}+\cdots & =0 \\
H_{1} \cos V_{1} & =\sin V_{1} \\
\cos H_{2} & =V_{2}
\end{aligned}
$$

The latter equations are well written under the harmonized forms. The same method can be directly applied to the inverse of a function and to the composite functions.

\section{CONCLUSION}

We have presented in this paper an extension of the dipolar method. Our aim is to reduce by one order of magnitude the simulation time of the main characteristics of the ultrastable quartz oscillators.

We have seen how to replace the Spice simulation by a symbolic calculation. This method leads to two difficulties: The treatment of the nonlinear components and the manipulation of very numerous coefficients.

We have given the set of equations that permit to manipulate symbolically the nonlinear terms including the standard functions, allowing thus to treat all the semiconductor components.

A direct application of the exposed equations in this paper leads to an exponential increase of the number of terms in the equations. An efficient method for manipulating these coefficients is currently being derived.

\section{REFERENCES}

[1] R. Brendel, D. Gillet, N. Ratier, F. Lardet-Vieudrin, and J. Delporte, "Nonlinear dipolar modelling of quartz crystal oscillators," in Proc. of 14th EFTF, 2000.

[2] M. Addouche, N. Ratier, D. Gillet, R. Brendel, F. Lardet-Vieudrin, and J. Delporte, "Adoq: A quartz crystal oscillators simulation software," in Proc. Int. Freq. Contr. Symp., 2001, pp. 753-757.

[3] M. Addouche, R. Brendel, D. Gillet, N. Ratier, F.Lardet-Vieudrin, and J. Delporte, "Modeling of quartz crystal oscillators by using nonlinear dipolar method," IEEE Trans. Ultrason., Ferroelect., Freq. Contr., vol. 50, no. 5, pp. 487-495, May 2003.

[4] Jiři Vlach and Kishore Singhal, Computer methods for circuit analysis and design, Van Nostrand Reinhold, second edition edition, 1994.

[5] Giuseppe Massobrio and Paolo Antognetti, Semiconductor Device Modeling with SPICE, MacGraw-Hill, Inc., second edition edition, 1993.

[6] Milton Abramowitz and Irene A. Stegun, Handbook of Mathematical Functions, Dover Publications, Inc., 1972. 